\title{
Subtle leakage of a Majorana mode into a quantum dot
}

\author{
E. Vernek, ${ }^{1,2}$ P. H. Penteado, ${ }^{2}$ A. C. Seridonio, ${ }^{3}$ and J. C. Egues ${ }^{2}$ \\ ${ }^{1}$ Instituto de Física, Universidade Federal de Uberlândia, Uberlândia, Minas Gerais 38400-902, Brazil \\ ${ }^{2}$ Instituto de Física de São Carlos, Universidade de São Paulo, São Carlos, São Paulo 13560-970, Brazil \\ ${ }^{3}$ Departamento de Física e Química, Universidade Estadual Paulista, Ilha Solteira, São Paulo 15385-000, Brazil
}

(Received 15 August 2013; revised manuscript received 10 April 2014; published 30 April 2014)

\begin{abstract}
We investigate quantum transport through a quantum dot connected to source and drain leads and side coupled to a topological superconducting nanowire (Kitaev chain) sustaining Majorana end modes. Using a recursive Green's-function approach, we determine the local density of states of the system and find that the end Majorana mode of the wire leaks into the dot, thus, emerging as a unique dot level pinned to the Fermi energy $\varepsilon_{F}$ of the leads. Surprisingly, this resonance pinning, resembling, in this sense, a "Kondo resonance," occurs even when the gate-controlled dot level $\varepsilon_{\text {dot }}\left(V_{g}\right)$ is far above or far below $\varepsilon_{F}$. The calculated conductance $G$ of the dot exhibits an unambiguous signature for the Majorana end mode of the wire: In essence, an off-resonance $\operatorname{dot}\left[\varepsilon_{\mathrm{dot}}\left(V_{g}\right) \neq \varepsilon_{F}\right]$, which should have $G=0$, shows, instead, a conductance $e^{2} / 2 h$ over a wide range of $V_{g}$ due to this pinned dot mode. Interestingly, this pinning effect only occurs when the dot level is coupled to a Majorana mode; ordinary fermionic modes (e.g., disorder) in the wire simply split and broaden (if a continuum) the dot level. We discuss experimental scenarios to probe Majorana modes in wires via these leaked/pinned dot modes.
\end{abstract}

DOI: 10.1103/PhysRevB.89.165314

PACS number(s): 71.10.Pm, 03.67.Lx, 74.25.F-, 74.45.+c

\section{INTRODUCTION}

Zero-bias anomalies in transport properties are one of the most intriguing features of the low-temperature physics in nanostructures. The canonical example is the zero-bias peak in the conductance of interacting quantum dots (QDs) coupled to metallic contacts, which is a clear manifestation of the Kondo effect [1,2] arising from the dynamical screening of the unpaired electron spin in the quantum dot by the itinerant electrons of the leads. Another example is the Andreev bound state arising from electron and hole scatterings at a normal-superconductor interface [3].

Recently, a new type of zero-bias anomaly has emerged in connection with the appearance of Majorana bound states in Zeeman-split nanowires with spin-orbit interaction in close proximity to an $s$-wave superconductor [4,5]. It is theoretically well established that these "topological" superconducting wires sustain chargeless zero-energy end states with peculiar features, such as braiding statistics, possibly relevant for topological quantum computation [6,7]. Experimentally, however, there is still controversy as to what the observed zero-bias peak really means: Kondo effect, Andreev bound states, and disorder effects are some of the possibilities [8-15]. Franz summarizes and discusses these issues in Ref. [16].

Here we propose a direct way to probe the Majorana end mode arising in a topological superconducting nanowire by measuring the two-terminal conductance $G$ through a dot side coupled to the wire, Figs. 1(a) and 1(b). Using an exact recursive Green's-function approach, we calculate the LDOS of the dot and wire and show that the Majorana end mode of the wire leaks into the dot [17], thus, giving rise to a Majorana resonance in the dot, Figs. 1(c) and 1(d). Surprisingly, we find that this dot-Majorana mode is pinned to the Fermi level $\varepsilon_{F}$ of the leads even when the gate-controlled dot level $\varepsilon_{\operatorname{dot}}\left(V_{g}\right)$ is far off-resonance $\varepsilon_{\text {dot }}\left(V_{g}\right) \neq \varepsilon_{F}$.

Based on the results above, we suggest three experimental ways for probing the Majorana end mode in the wire via the leaked/pinned Majorana mode in the dot: (i) with the dot kept off-resonance $\left[\varepsilon_{\operatorname{dot}}\left(V_{g}\right) \neq \varepsilon_{F}\right]$, one can measure $G$ vs $t_{0}$, the wire-dot coupling $t_{0}$ can be controlled by an external gate to see the emergence of the $e^{2} / 2 h$ peak in $G$ as the Majorana end mode leaks into the dot, Fig. 1(e) (cf. $\rho_{\text {dot }}$ and $\rho_{1}$, see also Fig. 2); (ii) alternatively, one can measure $G$ vs $V_{g}$ over a range in which $\varepsilon_{\text {dot }}\left(V_{g}\right)$ runs from far below to far above the Fermi level of the leads where we find $G$ to be essentially a plateau at $e^{2} / 2 h$, Figs. 1(f) and 1(g); (iii) yet another possibility is to drive the wire through a nontopological/topological phase transition, e.g., electrically via the spin-orbit coupling, temperature, or the chemical potential $\mu$ of the wire (Fig. 3), while measuring the conductance of the dot; the presence/absence of the Majorana end mode in the wire would drastically alter the conductance of the dot, see circles (black) and stars (green) in Fig. 1(g).

The above pinning of the dot-Majorana resonance at $\varepsilon_{F}$ is similar to that of the Kondo resonance [18]. However, the Kondo resonance only occurs for $\varepsilon_{\mathrm{dot}}\left(V_{g}\right)$ below $\varepsilon_{F}$ [cf. Figs. 1(h) and 1(i)] and yields a conductance peak at $e^{2} / h$ (per spin) instead. Even though there is no Kondo effect in our system (spinless dot), we conjecture that this symmetry of the dot-Majorana resonance with respect to $\varepsilon_{\mathrm{dot}}\left(V_{g}\right)$ above and below $\varepsilon_{F}$ could be used to distinguish Majorana-related peaks from those arising from the usual Kondo effect whenever this effect is relevant [19]. Moreover, this Majorana resonance in the dot follows quite simply by viewing the dot as an additional site (although with no pairing gap) of the Kitaev chain [20,21]. We emphasize that this unique pinning occurs only when the dot is coupled to a Majorana mode-a half-fermion state. When the dot is coupled to usual fermionic modes (bound, e.g., due to disorder, or not) in the wire, its energy level will simply split and will broaden as we discuss later on. A spin-full version of our model with a Hubbard $U$ interaction in the dot yields similar results [22].

The paper is organized as follows. In Secs. II and III, we present the Hamiltonian that describes our system and introduce the Majorana-Green's functions, respectively, that we use to calculate the relevant physical quantities. In Sec. IV, 

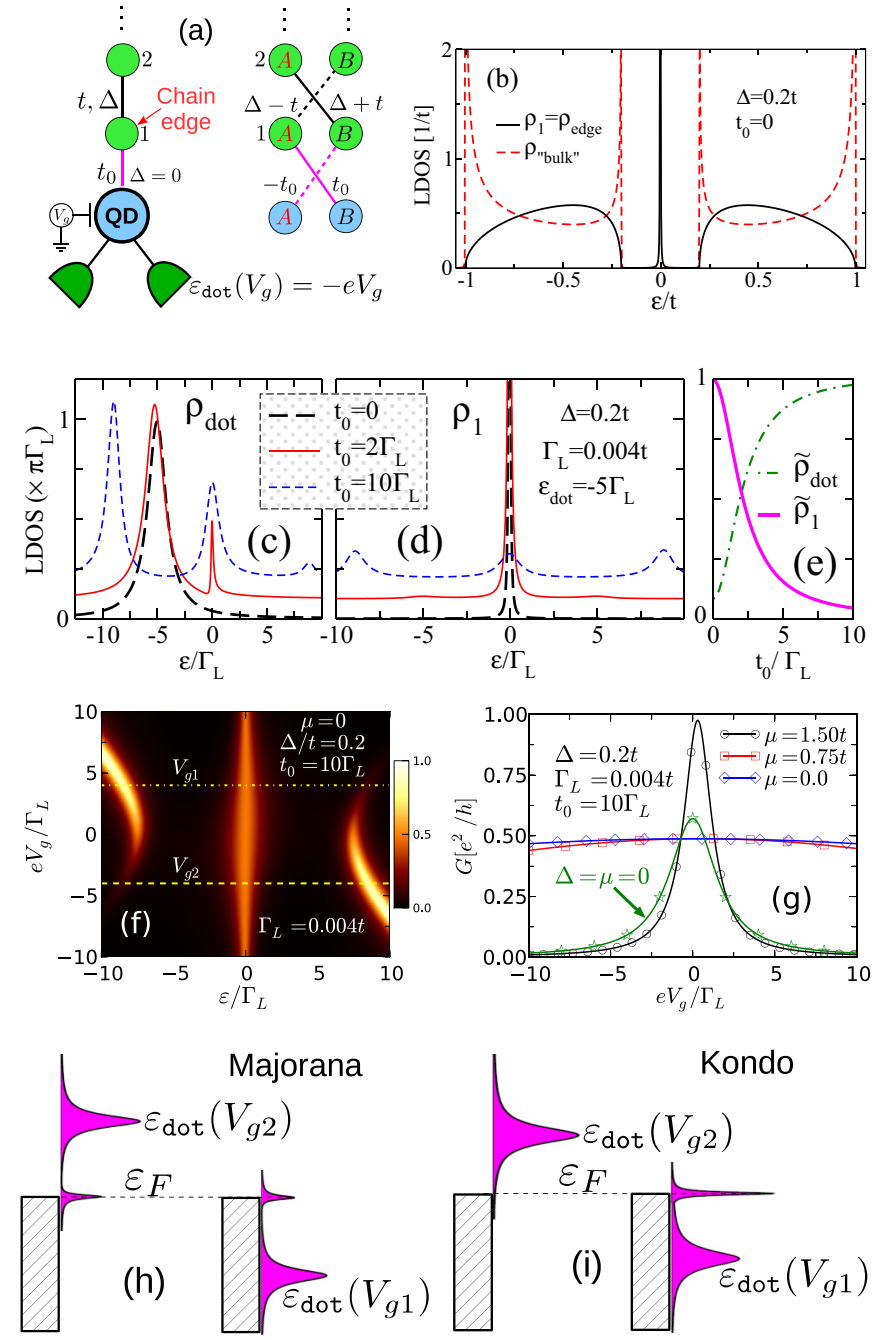

FIG. 1. (Color online) (a) Illustration of (left) a QD side coupled to a Kitaev wire and to two metallic leads and (right) the Majorana representation of the dot and the Kitaev chain. (b) "Bulk" [dashed (red) line] and edge [solid (black) line] chain local density of states (LDOS) for $t=10 \mathrm{meV}, \mu=0, \Delta=2 \mathrm{meV}, \Gamma_{L}=40 \mu \mathrm{eV}$, and $t_{0}=0$. (c) LDOS of the dot $\rho_{\text {dot }}$ and (d) of the first site of the Kitaev chain $\rho_{1}$ for the same set of parameters as in (b) and various values of $t_{0}$. For clarity, the curves in (c) and (d) are offset along the $y$ axis. (e) $\tilde{\rho}_{\mathrm{dot}}=\rho_{\mathrm{dot}}(0) / \rho_{\mathrm{dot}}^{\max }$ and $\tilde{\rho}_{1}=\rho_{\mathrm{dot}}(0) / \rho_{1}^{\max }$ at $\varepsilon=0$ as functions of $t_{0}$ in which $\rho_{\mathrm{dot}, 1}^{\max }=\max \left[\rho_{\mathrm{dot}, 1}\left(\varepsilon=0, t_{0}\right)\right]$. (f) Color map of the LDOS of the dot vs $\varepsilon$ and $e V_{g}$. (g) Conductance $G$ vs $e V_{g}$ for the same set of parameters as in (b) for various values of $\mu$. For comparison, we show the case $\Delta=\mu=0$ [stars (green)]. In (h) and (i), we sketch the LDOS of the dot for the Majorana and Kondo cases, respectively.

we present our numerical results and discussions. Finally, we summarize our main findings in Sec. V.

\section{MODEL HAMILTONIAN}

We consider a single-level spinless quantum dot coupled to two metallic leads and to a Kitaev chain [22], Fig. 1(a). To realize a single-level dot (spinless dot regime), we consider a dot with gate-controlled Zeeman-split levels $\varepsilon_{\text {dot }}^{\downarrow}\left(V_{g}\right)=$ $-e V_{g}(e>0)$ and $\varepsilon_{\mathrm{dot}}^{\uparrow}\left(V_{g}\right)=\varepsilon_{\mathrm{dot}}^{\downarrow}\left(V_{g}\right)+V_{Z}$ with $V_{Z}$ as the Zeeman energy. By varying $V_{g}$ such that $\left|e V_{g}\right|<V_{Z} / 2$, we can maintain the dot either empty [i.e., both spin-split levels above the Fermi level $\varepsilon_{F}$ (taken as zero) of the leads] or singly occupied [i.e., only one spin-split dot level, e.g., $\varepsilon_{\text {dot }}^{\downarrow}\left(V_{g}\right)$ below $\left.\varepsilon_{F}\right]$. This is the relevant spinless regime in our setup [23]. Typically [e.g., Fig. 1(g)], we vary $\left|e V_{g}\right|<10 \Gamma_{L}=0.4 \mathrm{meV}$, assuming a realistic Zeeman energy to attain topological superconductivity, i.e., $V_{Z} \simeq 0.8 \mathrm{meV}$ (see Rainis et al. [26]). This picture also holds true in the presence of a Hubbard $U$ term in the dot [22]). In this spinless regime, our Hamiltonian is $H=H_{\text {chain }}+H_{\text {dot }}+H_{\text {dot-chain }}+H_{\text {leads }}+H_{\text {dot-leads }}$, with $H_{\text {chain }}$ describing the chain,

$H_{\text {chain }}=-\mu \sum_{j=1}^{N} c_{j}^{\dagger} c_{j}-\frac{1}{2} \sum_{j=1}^{N-1}\left[t c_{j}^{\dagger} c_{j+1}+\Delta e^{i \phi} c_{j} c_{j+1}+\right.$ H.c. $]$,

$N$ is the number of chain sites, $c_{j}^{\dagger}\left(c_{j}\right)$ creates (annihilates) a spinless electron in the $j$ th site, and $\phi$ is an arbitrary phase. The parameters $t$ and $\Delta$ denote the intersite hopping and the superconductor pairing amplitude of the Kitaev model, respectively; its chemical potential is $\mu$.

The single-level dot Hamiltonian $H_{\mathrm{dot}}$ is

$$
H_{\mathrm{dot}}=\left(\varepsilon_{\mathrm{dot}}-\varepsilon_{F}\right) c_{0}^{\dagger} c_{0},
$$

where $c_{0}^{\dagger}\left(c_{0}\right)$ creates (annihilates) a spinless electron in the dot with energy $\varepsilon_{\mathrm{dot}}=-e V_{g}$ and $H_{\text {leads }}$ denotes the free-electron source $(S)$ and drain $(D)$ leads,

$$
H_{\text {leads }}=\sum_{\mathbf{k}, \ell=S, D}\left(\varepsilon_{\ell, \mathbf{k}}-\varepsilon_{F}\right) c_{\ell, \mathbf{k}}^{\dagger} c_{\ell, \mathbf{k}},
$$

where $c_{\ell, \mathbf{k}}^{\dagger}\left(c_{\ell, \mathbf{k}}\right)$ creates (annihilates) a spinless electron with wave vector $\mathbf{k}$ in the leads, whose Fermi level is $\varepsilon_{F}$. The couplings between the QD and the first site of the chain and between the QD and the leads are, respectively,

$$
H_{\text {dot-chain }}=t_{0}\left(c_{0}^{\dagger} c_{1}+c_{1}^{\dagger} c_{0}\right),
$$

and

$$
H_{\text {dot-leads }}=\sum_{\mathbf{k}, \ell=S, D}\left(V_{\ell, \mathbf{k}} c_{0}^{\dagger} c_{\ell, \mathbf{k}}+\text { H.c. }\right) .
$$

The quantity $V_{\ell, \mathbf{k}}$ is the tunneling between the QD and the source and drain leads, and $t_{0}$ is the hopping amplitude between the QD and the Kitaev chain.

\section{RECURSIVE GREEN'S FUNCTION AND LDOS}

Our model and approach are similar to those of Ref. [27] and go beyond low-energy effective Hamiltonians [28]. Let us introduce the Majorana fermions $\gamma_{\alpha j}, \alpha=A, B$, via $c_{j}=e^{-i \phi / 2}\left(\gamma_{B j}+i \gamma_{A j}\right) / 2$ and $c_{j}^{\dagger}=e^{i \phi / 2}\left(\gamma_{B j}-i \gamma_{A j}\right) / 2$, $j=0 \cdots N\left(j=0\right.$ is the dot) [20,29]. The $\gamma_{\alpha j}$ 's obey $\left[\gamma_{\alpha j}, \gamma_{\alpha^{\prime} j^{\prime}}\right]_{+}=2 \delta_{\alpha \alpha^{\prime}} \delta_{j j^{\prime}}$ and $\gamma_{\alpha j}^{\dagger}=\gamma_{\alpha j}$. We now define the Majorana-retarded Green's function,

$$
M_{\alpha i, \beta j}(\varepsilon)=-i \int_{-\infty}^{\infty} \Theta(\tau)\left\langle\left[\gamma_{\alpha i}(\tau), \gamma_{\beta j}(0)\right]_{+}\right\rangle e^{i \varepsilon(\tau)} d \tau,
$$

where $\langle\cdots\rangle$ denotes either a thermodynamic average or a ground-state expectation value at zero temperature, $\Theta(x)$ is 
the Heaviside function, and $\varepsilon \rightarrow \varepsilon+i \eta$ with $\eta \rightarrow 0^{+}$. We can express the electron Green's function as

$G_{i j}(\varepsilon)=\frac{1}{4}\left[M_{A i, A j}+M_{B i, B j}(\varepsilon)+i\left(M_{A i, B j}-M_{B i, A j}\right)\right]$,

and can determine the electronic $\operatorname{LDOS} \rho_{j}(\varepsilon)=(-1 / \pi)$ $\operatorname{Im} G_{j j}(\varepsilon)$,

$\rho_{j}(\varepsilon)=\frac{1}{4}\left[\mathcal{A}_{j}(\varepsilon)+\mathcal{B}_{j}(\varepsilon)-\frac{1}{\pi} \operatorname{Re}\left[M_{A j, B j}(\varepsilon)-M_{B j, A j}(\varepsilon)\right]\right]$.

In (8), we have introduced the Majorana $\operatorname{LDOS} \mathcal{A}_{j}(\varepsilon)=$ $(-1 / \pi) \operatorname{Im} M_{A j, A j}(\varepsilon)$ and $\mathcal{B}_{j}(\varepsilon)=(-1 / \pi) \operatorname{Im} M_{B j, B j}(\varepsilon)$.

Using the equation of motion for the Green's functions, we obtain a set of coupled matrix equations, e.g., for $j=0$ (dot),

$$
\mathbf{M}_{00}(\varepsilon)=\overline{\mathbf{m}}_{00}(\varepsilon)+\overline{\mathbf{m}}_{00}(\varepsilon) \mathbf{W}_{0}^{\dagger} \mathbf{M}_{10}(\varepsilon),
$$

where $\mathbf{M}_{i j}(\varepsilon)$ is [see Eq. (6)]

$$
\mathbf{M}_{i j}(\varepsilon)=\left[\begin{array}{ll}
M_{A i, A j}(\varepsilon) & M_{A i, B j}(\varepsilon) \\
M_{B i, A j}(\varepsilon) & M_{B i, B j}(\varepsilon)
\end{array}\right],
$$

$\overline{\mathbf{m}}_{j j}(\varepsilon)=\left[\mathbf{I}-\mathbf{m}_{j j}(\varepsilon) \mathbf{V}_{j}\right]^{-1} \mathbf{m}_{j j}(\varepsilon)$ and $\mathbf{m}_{j j}(\varepsilon)=2\left[\varepsilon-\Sigma_{0}\right.$ $\left.(\varepsilon) \delta_{0, j}\right]^{-1} \mathbf{I}$. Here $\Sigma_{0}(\varepsilon) \equiv \Sigma_{\text {dot }}=2 \sum_{\mathbf{k}}\left|\tilde{V}_{\mathbf{k}}\right|^{2}\left[\left(\varepsilon-\tilde{\varepsilon}_{\mathbf{k}}\right)^{-1}+\right.$ $\left.\left(\varepsilon+\tilde{\varepsilon}_{\mathbf{k}}\right)^{-1}\right]$ is the dot level broadening (leads) with $\tilde{\varepsilon}_{\mathbf{k}}=$ $\varepsilon_{\mathbf{k}}-\varepsilon_{F}, V_{S \mathbf{k}}=V_{D \mathbf{k}}=\tilde{V}_{\mathbf{k}} / \sqrt{2}$, and $\mathbf{I}$ as the $2 \times 2$ identity matrix. Finally,

$\mathbf{V}_{j}=\frac{1}{2}\left(\begin{array}{cc}0 & i \mu_{j} \\ -i \mu_{j} & 0\end{array}\right) \quad$ and $\quad \mathbf{W}_{j}=\frac{1}{2}\left[\begin{array}{cc}0 & i W_{j}^{(+)} \\ i W_{j}^{(-)} & 0\end{array}\right]$,

with $\mu_{0}=e V_{g}-2 \sum_{\mathbf{k}}\left|\tilde{V}_{\mathbf{k}}\right|^{2}\left[\left(\varepsilon-\tilde{\varepsilon}_{\mathbf{k}}\right)^{-1}-\left(\varepsilon+\tilde{\varepsilon}_{\mathbf{k}}\right)^{-1}\right], W_{0}^{( \pm)}$ $= \pm t_{0}$, and $\mu_{j}=\mu$ and $W_{j}^{( \pm)}=(\Delta \pm t) / 2$ for all $j>0$. The quantity $W_{j}^{( \pm)}$is an effective coupling matrix, see Fig. 1(a). In the wideband limit and assuming a constant $\tilde{V}_{\mathbf{k}}=\sqrt{2} \tilde{V}$, we obtain $\Sigma_{\text {dot }}(\varepsilon)=-2 i \Gamma_{L}$ and $\mu_{0}=e V_{g}=-\varepsilon_{\text {dot }}$ with the broadening $\Gamma_{L}=2 \pi|\tilde{V}|^{2} \rho_{L}$ and $\rho_{L}=\rho\left(\varepsilon_{F}\right)$ being the DOS of the leads. Similar to (9), we find, for the first site $(j=1)$ of the chain,

$$
\mathbf{M}_{11}(\varepsilon)=\tilde{\mathbf{m}}_{11}(\varepsilon)+\tilde{\mathbf{m}}_{11}(\varepsilon) \mathbf{W}_{1}^{\dagger} \mathbf{M}_{21}(\varepsilon),
$$

with $\tilde{\mathbf{m}}_{11}(\varepsilon)=\left[\mathbf{I}-\overline{\mathbf{m}}_{11}(\varepsilon) \mathbf{W}_{0} \overline{\mathbf{m}}_{00}(\varepsilon) \mathbf{W}_{0}^{\dagger}\right]^{-1} \overline{\mathbf{m}}_{11}(\varepsilon)$. We can then recursively obtain the Majorana matrix at any site.

\section{NUMERICAL RESULTS}

Following realistic simulations [26,30] and experiments [8], here we assume $t=10 \mathrm{meV}$, the dot level broadening $\Gamma_{L}=$ $4.0 \times 10^{-3} t=40 \mu \mathrm{eV}$ and set $\varepsilon_{F}=0$ (we also set $\phi=0$ ). In Fig. 1(b), we show the LDOS as a function of the energy $\varepsilon$ for a site in the middle and on the edge of the chain $\rho_{\text {bulk }}$ [dashed (red) curve] and $\rho_{1}=\rho_{\text {edge }}$ [solid (black) curve], respectively, for $t_{0}=0$ (decoupled chain) and $\Delta=0.2 t=2 \mathrm{meV}$. Note that $\rho_{\text {bulk }}$ is fully gapped, whereas, $\rho_{1}=\rho_{\text {edge }}$ exhibits a midgap zero-energy peak, corresponding to the end Majorana state of the chain.

Figures 1(c) and 1(d) show the LDOS of the dot $\rho_{\text {dot }}$ and of the first chain site $\rho_{1}$ as functions of $\varepsilon$ for $\varepsilon_{\text {dot }}=-5 \Gamma_{L}$ and three different values of $t_{0}$. For clarity, the curves are offset vertically. For $t_{0}=0$ [long dashed (black) line], we see just the usual single-particle peak of width $\Gamma_{L}$ centered at $\varepsilon=\varepsilon_{\mathrm{dot}}$. Observe that there is essentially no density of states at $\varepsilon=0$ since the dot level is far below the Fermi level of the leads. As we increase $t_{0}$ to $2 \Gamma_{L}$ [fine solid (red) line], however, we observe the emergence of a sharp peak at $\varepsilon=0$ in addition to the peak at $\varepsilon \approx \varepsilon_{\text {dot }}$. For $t_{0}=10 \Gamma_{L}$ [dashed (blue) line in Fig. 1(c)], the single-particle peak in $\rho_{\text {dot }}$ slightly moves to lower energies, while its zero-energy peak increases to 0.5 (in units of $\pi \Gamma_{L}$ ). As this peak appears in $\rho_{\text {dot }}$ for increasing $t_{0}$ 's, the Majorana central peak in Fig. 1(d) decreases. We can still see a peak in $\rho_{1}$ for $t_{0}=10 \Gamma_{L}$, dashed (blue) line in Fig. 1(d), but much weaker than its $t_{0}=0$ value. We further show $\quad \tilde{\rho}_{\mathrm{dot}}=\rho_{\mathrm{dot}}(0) / \rho_{\mathrm{dot}}^{\max } \quad$ and $\quad \tilde{\rho}_{1}=\rho_{1}(0) / \rho_{1}^{\max }, \rho_{\mathrm{dot}, 1}^{\max }=$ $\max \left[\rho_{\mathrm{dot}, 1}\left(\varepsilon=0, t_{0}\right)\right]$ vs $t_{0}$ in Fig. $1(\mathrm{e})$, clearly showing the wire Majorana leakage into the dot.

In Fig. 1(f), we display a color map of the electronic LDOS $\rho_{\text {dot }}$ vs $\varepsilon$ and $e V_{g}$ for the wire in the topological phase $(\Delta>0$ and $|\mu|<t)$ with $\mu=0$. At $e V_{g}=0$, we see three peaks of $\rho_{\text {dot }}$ vs $\varepsilon$, similar to those of Fig. 2 of Ref. [27]. In contrast, by fixing $\varepsilon=0$ and varying $e V_{g}$, we see that the zero-energy peak remains essentially unchanged over the range of $e V_{g}$ shown. More strikingly, this central peak is pinned at $\varepsilon=\varepsilon_{F}=0$ for $e V_{g}>0$ and $e V_{g}<0$. The pinning for $\varepsilon_{\mathrm{dot}}$ below $\varepsilon_{F}=0$ is similar to that of the Kondo resonance, which, however, is known to occur at $\pi \Gamma_{L}$, cf. Figs. 1(h) and 1(i).

Here again, one can measure $G$ vs $V_{g}$ [Fig. $\left.1(\mathrm{~g})\right]$ : For the wire in its trivial phase $(|\mu|>t)$, e.g., $\mu=1.5 t$ [circles (black)], $G$ exhibits a single peak, whose maximum corresponds to $\varepsilon_{\text {dot }}\left(V_{g}\right)$ crossing the Fermi level. Note that the peak is not at $e V_{g}=0$ but slightly shifted. This arises from the small real part of the self-energy in the dot Green's function. In the topological phase $(|\mu|<t)$, e.g., $\mu=0$ and $\mu=0.75 t$ [squares (red) and diamonds (blue), respectively], we see an almost constant $G \simeq e^{2} / 2 h$ for $e V_{g}$ up to $\pm 10 \Gamma_{L}$. This conductance plateau is similar to that produced by the Kondo resonance [1], except that here $G$ is half of it (per spin) and the plateau occurs even for $\varepsilon_{\mathrm{dot}}>\varepsilon_{F}$.

The Majorana LDOS $\mathcal{A}_{\mathrm{dot}}$ and $\mathcal{B}_{\text {dot }}$ shown in Figs. 2(a) and 2(b), respectively, as functions of $\varepsilon$ and $e V_{g}$ [same

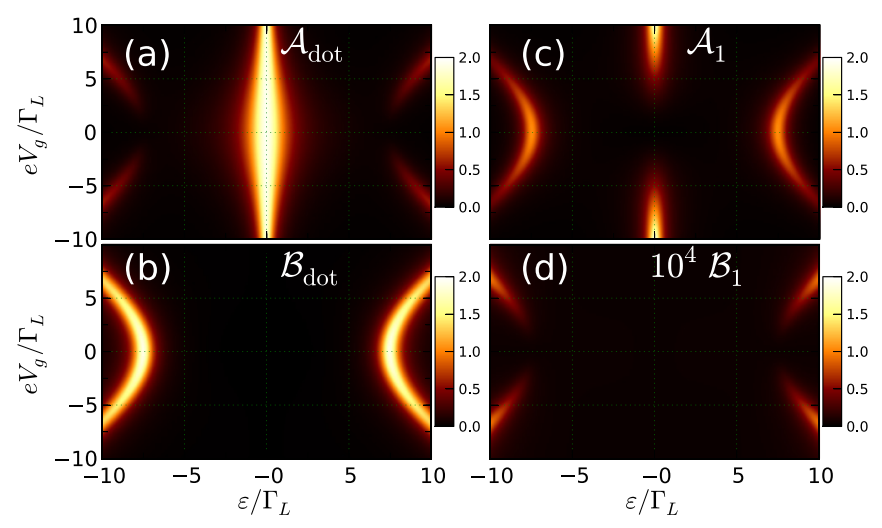

FIG. 2. (Color online) Color map of the local density of states for Majoranas "A" (top) and "B" (bottom) at the dot (left) and at the first site of the chain (right) as a function of $\varepsilon$ and $e V_{g}$ for $t=$ $10 \mathrm{meV}, \Delta=0.2 t, \Gamma_{L}=40 \mu \mathrm{eV}, t_{0}=10 \Gamma_{L}$, and $\mu=0$. Panel (d) shows $10^{4} \mathcal{B}_{1}$ 


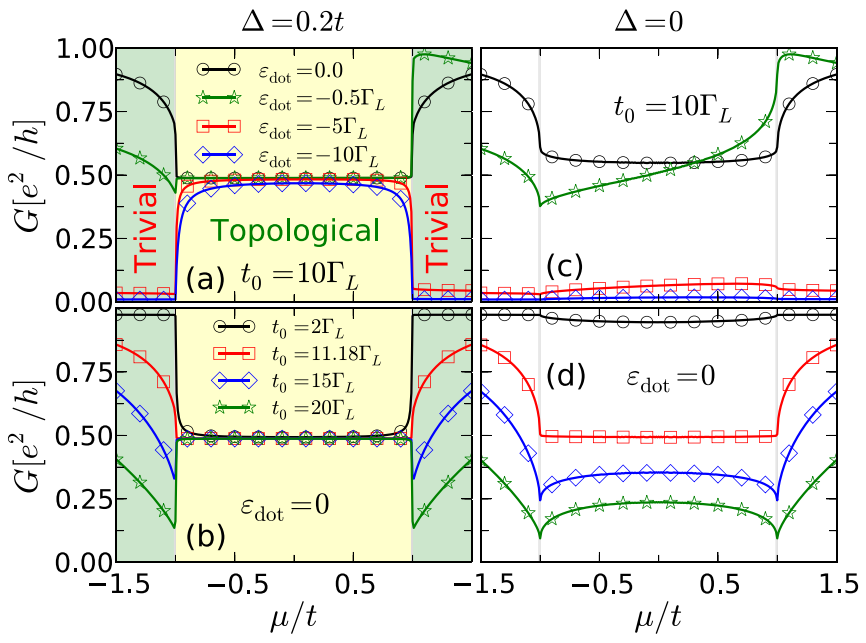

FIG. 3. (Color online) Conductance $G$ as a function of $\mu$ for $t=$ $10 \mathrm{meV}, \Delta=0.2 \mathrm{meV}$, and (a) $t_{0}=10 \Gamma_{L}$ and different values of $\varepsilon_{\mathrm{dot}}$ and (b) $\varepsilon_{\mathrm{dot}}=0$ and distinct $t_{0}$ 's. The lighter (yellow) and darker (green) regions in (a) and (b) highlight the topological $(|\mu|<t)$ and trivial $(|\mu|>t)$ phases of the chain, respectively. Panels (c) and (d) correspond to (a) and (b), respectively, but for $\Delta=0$.

parameters as in Fig. 1(f)], display a zero-energy peak in $\mathcal{A}_{\text {dot }}$ and none in $\mathcal{B}_{\text {dot }}$. This shows that the pinned dot-Majorana peak in Fig. 1(f) arises from the Majorana A only. We note that the peaks in $\mathcal{B}_{\text {dot }}$ at $\varepsilon \approx \pm 7 \Gamma_{L}$ (for $e V_{g}=0$ ) are affected by the dot-wire Majorana coupling as compared to the $\Delta=t$ case. For couplings to any ordinary fermionic wire modes, the dot LDOS would obey $\mathcal{A}_{\text {dot }}=\mathcal{B}_{\text {dot }}$, and it would split and would broaden.

Figures 2(c) and 2(d) show that the Majorana LDOS of the first chain site $\mathcal{A}_{1}$ and $\mathcal{B}_{1}$ have no zero-energy peaks, thus, indicating that the wire end mode has, indeed, leaked into the dot. We see two peaks in $\mathcal{A}_{1}$ at $\varepsilon= \pm 7 \Gamma_{L}$ [see Fig. 2(b)] resulting from the coupling $\sim t_{0}$ between $A_{1}$ and $B_{\text {dot }}$; see Fig. 1(a). A careful look at Fig. 2(c) reveals an enhancement of the zero-energy peaks for $e V_{g} \gtrsim 5 \Gamma_{L}$ as a result of the coupling between the dot Majorana $\mathrm{A}$ and the Majoranas of the chain via a finite $\varepsilon_{\mathrm{dot}}$. The strength of this peak is much smaller than its magnitude without the dot.

Figure 3(a) shows the conductance $G$ vs $\mu$ for several $\varepsilon_{\text {dot }}$ 's [same parameters as in Figs. 1(f) and 1(g)]. For $\varepsilon_{\text {dot }}=0$ [circles (black)] and $|\mu|>t$ (trivial phase), $G$ arises from the single-particle dot level at $\varepsilon_{F}$. The effect of the chain is essentially to shift and broaden $\varepsilon_{\text {dot }}$ so that the value $e^{2} / h$ is reached only for $|\mu| \gg t$. As $\mu$ varies across $\pm t$, the wire undergoes a trivial-to-topological transition, and $G$ suddenly decreases to $e^{2} / 2 h$ as the leaked dot Majorana appears. For $\varepsilon_{\mathrm{dot}} \neq 0$, the asymptotic $(|\mu| \gg t)$ value of $G$ is no longer $e^{2} / h$ as $\varepsilon_{\text {dot }}$ cannot attain $\varepsilon_{F}$. The squares (red) and diamonds (blue) in Fig. 3(a) show a tiny conductance for $\mu>t$. However, as $|\mu|$ becomes smaller than $t$, both curves rapidly go to $e^{2} / 2 h$.

In Fig. 3(b), we fix $\varepsilon_{\text {dot }}=0$ and plot the conductance $G$ as a function of $\mu$ for distinct $t_{0}$ 's. As $t_{0}$ increases, $G$ remains pinned at $e^{2} / 2 h$ in the topological regime, whereas, it decreases in the trivial phase since the dot level shifts due to the chain self-energy $\sim t_{0}^{2}$. Figures 3(c) and 3(d) show $G$ for $\Delta=0$ and the same parameters as in Figs. 3(a) and 3(b), respectively. For $|\mu|<t, G$ is very sensitive to $\varepsilon_{\mathrm{dot}}$ for a fixed $t_{0}=10 \Gamma_{L}$ [Fig. 3(c)] and to $t_{0}$ for $\varepsilon_{\text {dot }}=0$ [Fig. 3(d)], which contrasts with its practically constant value for $\Delta=0.2 t$, Figs. 3(a) and 3(b). This is so because the wire acts as a third normal lead for $\Delta=0$ and $t_{0} \neq 0$, so the source drain $G$, e.g., for $\mu=0$, reduces to $G=\left(e^{2} / h\right) \Gamma_{L} /\left(\Gamma_{L}+\Gamma_{\text {chain }}\right)$, where $\Gamma_{\text {chain }}=2 t_{0}^{2} / t$ is the broadening due to the chain [31]. Curiously, for $t_{0}=$ $11.18 \Gamma_{L}$ and $\varepsilon_{\mathrm{dot}}=0$, the $G$ curves are indistinguishable for $\Delta=0$ and $\Delta \neq 0$, being pinned at $e^{2} / 2 h$ in the topological and trivial phases, cf. squares in Figs. 3(d) and 3(c). Therefore, the peak value $G=e^{2} / 2 h$, first found in Ref. [27] in a similar setup as ours but only for an on-resonance dot (i.e., $\varepsilon_{\mathrm{dot}}=0=\varepsilon_{F}$ ), is not per se a "smoking-gun" evidence for a Majorana end mode in conductance measurements as we find that this peak value can appear even in the trivial phase of the wire. One should vary, e.g., $\varepsilon_{\mathrm{dot}}$ and/or $t_{0}$ to tell these phases apart as we do in Fig. 3. Finally, the kinks in Fig. 3(d) [e.g., diamonds (blue) and stars (green)] result from discontinuities in $\Sigma_{\text {chain }}$ [31] at $\mu= \pm t$.

\section{CONCLUDING REMARKS}

We have used an exact recursive Green's-function approach to calculate the LDOS and the two-terminal conductance $G$ through a quantum dot side coupled to a Kitaev wire. Interestingly, we found that the end Majorana mode of the wire leaks into the quantum dot, thus, originating a resonance pinned to the Fermi level of the leads $\varepsilon_{F}$. In contrast to the usual Kondo resonance arising only for $\varepsilon_{\text {dot }}$ below $\varepsilon_{F}$, this unique dot-Majorana resonance appears pinned to $\varepsilon_{F}$ even when the gate-controlled energy level $\varepsilon_{\text {dot }}\left(V_{g}\right)$ is far above or below $\varepsilon_{F}$, provided that the wire is in its topological phase. This leaked Majorana dot mode provides a clear-cut way to probe the Majorana mode of the wire via conductance measurements through the dot.

\section{ACKNOWLEDGMENTS}

We acknowledge helpful discussions with F. M. Souza. J.C.E. also acknowledges valuable discussions with D. Rainis. This work was supported by the Brazilian agencies $\mathrm{CNPq}$, CAPES, FAPESP, FAPEMIG, and PRP/USP within the Research Support Center Initiative (NAP Q-NANO).
[1] D. Goldhaber-Gordon, H. Shtrikman, D. Mahalu, D. AbuschMagder, U. Meirav, and M. A. Kastner, Nature (London) 391, 156 (1998).

[2] S. M. Cronenwett, T. H. Oosterkamp, and L. P. Kouwenhoven, Science 281, 540 (1998).

[3] A. A. Golubov, A. Brinkman, Y. Tanaka, I. I. Mazin, and O. V. Dolgov, Phys. Rev. Lett. 103, 077003 (2009).
[4] R. M. Lutchyn, J. D. Sau, and S. Das Sarma, Phys. Rev. Lett. 105, 077001 (2010).

[5] Y. Oreg, G. Refael, and F. von Oppen, Phys. Rev. Lett. 105, 177002 (2010).

[6] A. Y. Kitaev, Ann. Phys. 303, 2 (2003).

[7] C. Nayak, S. H. Simon, A. Stern, M. Freedman, and S. Das Sarma, Rev. Mod. Phys. 80, 1083 (2008). 
[8] V. Mourik, K. Zuo, S. M. Frolov, S. R. Plissard, E. P. A. M. Bakkers, and L. P. Kouwenhoven, Science 336, 1003 (2012).

[9] M. T. Deng, C. L. Yu, G. Y. Huang, M. Larsson, P. Caroff, and H. Q. Xu, Nano Lett. 12, 6414 (2012).

[10] A. Das, Y. Most, Y. Oreg, M. Heiblum, and H. Shtrikman, Nat. Phys. 8, 887 (2012).

[11] E. J. H. Lee, X. Jiang, R. Aguado, G. Katsaros, C. M. Lieber, and S. De Franceschi, Phys. Rev. Lett. 109, 186802 (2012).

[12] A. D. K. Finck, D. J. Van Harlingen, P. K. Mohseni, K. Jung, and X. Li, Phys. Rev. Lett. 110, 126406 (2013).

[13] K. T. Law, P. A. Lee, and T. K. Ng, Phys. Rev. Lett. 103, 237001 (2009).

[14] D. I. Pikulin, J. P. Dahlhaus, M. Wimmer, H. Schomerus, and C. W. J. Beenakker, New J. Phys. 14, 125011 (2012).

[15] H. O. H. Churchill, V. Fatemi, K. Grove-Rasmussen, M. T. Deng, P. Caroff, H. Q. Xu, and C. M. Marcus, Phys. Rev. B 87, 241401 (2013).

[16] M. Franz, Nat. Nanotechnol. 8, 149 (2013).

[17] Interestingly, J. Klinovaja and D. Loss, Phys. Rev. B 86, 085408 (2012) have reported Majorana modes leaking across a superconducting-normal interface. See also D. Chevallier, D. Sticlet, P. Simon, and C. Bena, ibid. 85, 235307 (2012).

[18] A. C. Hewson, in The Kondo Problem to Heavy Fermions, edited by D. Edwards and D. Melville, Cambridge Studies in Magnetism (Cambridge University Press, Cambridge, UK, 1993).

[19] When the Kondo resonance coexists with the dot-Majorana mode, the total conductance through the dot should ideally attain $3 e^{2} / 2 h\left[e^{2} / h\right.$ (due to Kondo) $+e^{2} / 2 h$ (Majorana) $]$ as was found by M. Lee, J. S. Lim, and R. López, Phys. Rev. B 87, 241402 (2013) for a dot in the resonant-tunneling regime. See also Cheng et al., arXiv:1308.4156 and A. Golub, I. Kuzmenko, and Y. Avishai, Phys. Rev. Lett. 107, 176802 (2011) for a discussion on the interplay between Kondo- and Majorana-induced couplings in a quantum dot.
[20] A. Y. Kitaev, Phys.-Usp. 44, 131 (2001).

[21] For an interesting proposal for the realization of the Kitaev chain, see I. C. Fulga, A. Haim, A. R. Akhmerov, and Y. Oreg, New J. Phys. 15, 045020 (2013).

[22] We have also implemented a spin-full version of our system, considering a tight-binding nanowire + Rashba spin-orbit interaction + proximity-induced superconductivity + a Zeeman field (a wire with these ingredients can be mapped onto the Kitaev chain [24,25]), which corroborates our findings. In addition, we have considered a Hubbard $U$ term in the dot and have verified that our results still hold, provided that only a single spin-split dot level lies below the Fermi level of the leads (i.e., Coulomb blockade is irrelevant in this regime). These results will be described elsewhere.

[23] Interestingly, this regime (empty or singly occupied dot) should, by itself, prevent the Kondo effect and Andreev bound states in our setup. Andreev bound states, however, have a strong gate voltage dependence as reported by R. S. Deacon, Y. Tanaka, A. Oiwa, R. Sakano, K. Yoshida, K. Shibata, K. Hirakawa, and S. Tarucha, Phys. Rev. Lett. 104, 076805 (2010) and appear (mostly) as doublets not pinned to $\varepsilon_{F}$ [see also E. J. H. Lee et al., Nat. Nanotechnol. 9, 79 (2014)].

[24] J. Alicea, Phys. Rev. B 81, 125318 (2010).

[25] J. Alicea, Y. Oreg, G. Refael, F. von Oppen, and M. P. A. Fisher, Nat. Phys. 7, 412 (2011).

[26] D. Rainis, L. Trifunovic, J. Klinovaja, and D. Loss, Phys. Rev. B 87, 024515 (2013).

[27] D. E. Liu and H. U. Baranger, Phys. Rev. B 84, 201308 (2011).

[28] M. Leijnse and K. Flensberg, Phys. Rev. B 84, 140501 (2011).

[29] J. Alicea, Rep. Prog. Phys. 75, 076501 (2012).

[30] E. Prada, P. San-Jose, and R. Aguado, Phys. Rev. B 86, 180503(R) (2012).

[31] The general expression for the conductance at $T=0$ is $G=\Gamma_{L}\left(\Gamma_{L}-\operatorname{Im} \Sigma_{\text {chain }}\right) /\left[\left(\operatorname{Re} \Sigma_{\text {chain }}\right)^{2}+\left(\Gamma_{L}-\operatorname{Im} \Sigma_{\text {chain }}\right)^{2}\right]$ with $\Sigma_{\text {chain }}=2 t_{0}^{2}\left(1-\sqrt{1-g_{0}^{2} t^{2}}\right) / g_{0} t^{2}$ and $g_{0}=(-\mu+i \eta)^{-1}$. 